\title{
Corela
}

Cognition, représentation, langage

HS-1 1 | 2012

RJC Cotexte, contexte, situation

\section{Les noms de rues : lieux d'une langue nationale}

\section{Carolina Fedatto}

\section{OpenEdition}

\section{Journals}

Édition électronique

URL : http://journals.openedition.org/corela/2204

DOI : $10.4000 /$ corela.2204

ISSN : 1638-573X

\section{Éditeur}

Cercle linguistique du Centre et de l'Ouest - CerLICO

\section{Référence électronique}

Carolina Fedatto, «Les noms de rues : lieux d'une langue nationale », Corela [En ligne], HS-11 | 2012 mis en ligne le 02 avril 2012, consulté le 21 avril 2019. URL : http://journals.openedition.org/ corela/2204 ; DOI : 10.4000/corela.2204

Ce document a été généré automatiquement le 21 avril 2019

\section{(c) (i) (2)(2)}

Corela - cognition, représentation, langage est mis à disposition selon les termes de la licence Creative Commons Attribution - Pas d'Utilisation Commerciale - Partage dans les Mêmes Conditions 4.0 International. 


\title{
Les noms de rues : lieux d'une langue nationale
}

\author{
Carolina Fedatto
}

\section{Introduction}

1 Les inscriptions urbaines ${ }^{1}$ sont des lieux singuliers pour étudier les effets de la mémoire (et de l'oubli) dans l'histoire d'une langue et d'un espace national. L'histoire des signes urbains (odonymes, architecture) n'est pas un héritage passif que l'actualité maintient ou ruine, ces formes symboliques sont l'effet du travail des formes-sujet-historiques ${ }^{2}$ qui les fabriquèrent aussi bien que des changements dans les idéologies alors dominants.

Dans cet article, nous nous attacherons spécifiquement à l'étude diachronique des noms de rue, à leurs changements de forme et de sens. La présence et la modification des noms de rue attestent d'une part, chez les habitants, le besoin de mieux identifier des espaces urbains, d'autre part, chez les citoyens, l'affirmation d'un espace national. Cette problématique, issue de deux axes de recherche développés à l'Université de Campinas (Brésil) : « Histoire des Idées Linguistiques au Brésil $»^{3}$ et "Savoir Urbain et Langage $»^{4}$, permet de poser le rôle de l'urbanisation dans le processus de construction d'une langue nationale et son espace.

L'imaginaire de localisation que les odonymes urbains construisent est parallèle à l'apparente stabilité de la langue nationale. Ces deux phénomènes sont parties intégrantes du même fait politique: l'identification, par l'Etat, du sujet en citoyen (Orlandi, $2001: 104$ ), c'est-a-dire en un individu qui doit savoir où il marche, être sûr de la langue qu'il parle et connaître ses origines et traditions. Le sujet moderne est constitué par une injonction de droits et de devoirs ; il n'est plus, comme au Moyen-âge, interpelé par un principe d'obéissance à Dieu. La constitution des Etats-nations marque aussi et en même temps la constitution d'un sujet libre et soumis. ${ }^{5}$ L'espace et son histoire sont donc constitués de matérialités qui font sens pour le chercheur qui veut analyser parallèlement 
ce processus d'identification du sujet en tant que citoyen et la stabilisation de la langue nationale.

4 Nous nous appuierons pour cela sur le rapport entre l'histoire de l'urbanisation et la construction du savoir sur les langues étudié par Auroux (1989). Selon lui, les deux grandes révolutions scientifiques de l'histoire de l'humanité, l'invention de l'écriture (3.000 avant notre ère) et la construction savante des savoirs de la langue avec la grammatisation massive des langues du monde (XVIe siècle), eurent de forts liens avec les injonctions politico-administratives imposées par les villes anciennes, par la formation des Etats-nations et la naissance de nouvelles villes dans les territoires conquis par les Européens dès le XVIe siècle.

5 A partir du jeu entre l'inscription d'un sens dans l'histoire de la ville et sa déstabilisation et, à travers l'analyse de la matérialité linguistico-historique des noms de rue, nous proposons une compréhension de ce qu'on pourrait appeler une "textualité urbaine brésilienne ", c'est-a-dire un ensemble de productions écrites contraintes par un espace urbain. Nous distinguons d'abord dans notre étude trois moments importants du rapport nomination-urbanisation au Brésil. Le premier correspond à l'installation en 1587 de l' Igreja de São Francisco dans la ville de Salvador, capitale coloniale brésilienne, et à son implication dans le début du processus de colonisation linguistique. Le deuxième correspond au déplacement de la Bibliothèque Nationale au Brésil en même temps que le roi du Portugal en 1808 et concentre donc les signes de l'ambivalence dépendance/ indépendance de la métropole. Le troisième est la création en 1874 du Colégio Culto à Ciência de Campinas comme un espace de production du savoir sur la langue portugaise au Brésil, une marque à la fois d'affirmation et de résistance d'une langue nationale et de sa théorisation.

De façon générale, les noms de rue peuvent être considérés comme les produits d'un choix linguistique et comme des «indicateurs» de l'histoire politique d'un pays. Ces inscriptions urbaines localisent soit le trajet immédiat du sujet dans les chemins de la cité soit les savoirs accumulés dans les débats sur la mémoire linguistique dans le champ de l'urbain. Notre hypothèse est que ces trois moments de l'histoire politique brésilienne (colonie, empire et république) sont d'une part, représentés dans l'histoire de ces trois institutions (l'église, la bibliothèque, le collège) et d'autre part, matérialisés dans le changement (ou la permanence) des noms de rue. Nous considérons que l'étude de ces trois lieux en même temps que l'analyse de leurs noms peuvent fortement contribuer à la compréhension du lien existant entre langue et territoire dans la mesure où les lieux, d'un côté, leurs dénominations, de l'autre, incarnent et expriment les transitions historiques brésiliennes spécifiques des processus de colonisation.

7 Dans un premier temps, nous tenterons de penser la dénomination de ces points de référence : nous nous demanderons à quoi réfère l'histoire de ses changements; ensuite nous interrogerons les relations qu'elles établissent avec la société (usages, accès, statuts); enfin nous réfléchirons au jeu de forces qui soutiennent leur apparition mais aussi peuvent expliquer leur déstructuration ou leur effacement. Toutefois, il convient auparavant de préciser les positions théoriques à la base de ce travail. 


\section{Fondements théoriques} dans en territoires de colonisation et dans le contexte linguistique de la constitution d'une langue nationale pour ces territoires en construction. La contradiction historique et l'équivoque du langage ${ }^{6}$, en tant que réels (on ne peut empêcher leurs effets sur le mouvement de signification), sont confrontées au besoin imaginaire de légitimer la nation à travers l'unité du territoire et de la langue. elles constituent des points de référence. On peut définir le point de référence comme un lieu dans l'espace urbain doublement affecté par la temporalité. Du côté synchronique, un point de référence est construit par l'imaginaire de localisation (quand on est ici on croit savoir où on est) et, du côté de la diachronie, il inaugure un imaginaire de rétrospection (son existence est un souvenir du temps dans l'espace; marque d'une altérité). Ces deux formes du temps dans l'espace sont de l'ordre de l'imaginaire.

Du point de vue théorico-analytique, la construction d'un point de référence dans l'espace peut être abordée à partir de la discussion d'au moins deux thèmes forts en sciences du langage : le processus de mise en texte et la construction du référent par le langage.

11 D'un côté, on considère que le mouvement même de construction des villes est un processus de mise en texte (Orlandi, $2001: 42$ et $64 \mathrm{sq}$.), un ensemble, avec son effet d'unité, qui produit et expose une trajectoire interprétative pour l'inscription des sujets et des sens dans l'espace urbain. Texte plein de reformulations : l'inauguration d'un bâtiment, l'institution de ses fonctions, le remplacement d'un nom par un autre, les changements d'adresse et les réformes sont des gestes triviaux, quotidiens, mais non sans importance pour la constitution d'une unité imaginaire pour l'espace urbain. Ces gestes d'écriture des villes produisent un savoir dans le quotidien. Une accumulation de représentations, de connaissances infléchit le savoir et les modes de vie des sujets. Le sujet, sa langue et ses traditions restent marqués par l'inauguration d'institutions et par la dénomination des éléments constitutifs de l'espace urbain. Qu'il soit dedans ou dehors, le citoyen sera nécessairement constitué à son tour par ces espaces, par les savoirs qui se matérialisent dans l'architecture, dans la dénomination, dans les discours inauguraux, etc. Quels sont les savoirs qui errent au hasard des rues? La localisation des constructions, les noms de ses rues et leur rôle social seront nos points de départ pour discuter les processus de mise en texte des références sur la langue dans l'histoire de la ville au Brésil.

D'un autre côté, la question de la dénomination touche une problématique d'intérêt sémantico-énonciatif qui suspend le rapport au langage de la référence, du monde et des choses. Les sémanticiens prennent toujours en compte le fait que le langage " parle » de quelque chose et que cette chose est désormais construite dans le langage (Guimarães, 2005 : 32). On peut dire donc que la production d'une référence nominale dans et par l'espace urbain est attachée à la symbolisation de cet espace: le rapport établi par le langage avec d'autres constructions, avec l'occupation des villes, avec les besoins politiques de construire une origine, un passé et une tradition. Le principe d'extériorité de la référence implique la possibilité de se demander à quoi le nom réfère et conduit à une réflexion sur le processus imaginaire de production de la réalité. 
13 La langue est un système relatif (à l'histoire) ${ }^{9}$ de formes qui nous écartent de l'existence naturelle des choses. On ne peut pas tout nommer ni tout dire. Mais l'illusion de complétude est une condition de possibilité du dire. On risquerait sinon de glisser dans l'abîme des formes paradigmatiques substituables potentiellement à ce qui a été dit ${ }^{10}$. En fait, il y a deux mécanismes pour freiner cette chute dans l'infini-autre. Le premier est la langue elle-même avec sa matérialité qui nous oblige à dire les choses de telle ou telle façon ${ }^{11}$. Le seconde est l'histoire qui produit à la fois le mouvement vers de nouveaux sens et limite les possibilités d'interprétation des événements linguistiques ${ }^{12}$.

Le sujet de langage ne peut pas modifier le réel ou y accéder par sa volonté : le langage (le symbolique) est la seule façon de s'y confronter en même temps que le rapport entre le système linguistique et le réel modifie à la fois la langue et les moyens d'appréhension du monde. La langue nous confronte au réel, à l'impossible de n'être pas comme cela, comme cela est (Pêcheux, 1983, p. 29). Le propre du rapport entre le réel et le symbolique est l'interférence mutuelle: le monde pose des événements à comprendre et la langue impose des conditions pour cette interprétation. En considérant donc que le langage a son opacité et que les référents sont signifiés par la matérialité linguistique et ne lui préexiste pas à côté d'elle mais qu'ils sont toujours dotes de sens, nous nous demandons comment les noms produisent un effet de localisation, non seulement dans l'espace, mais dans la confluence de plusieurs sens, selon l'imaginaire d'un point de repère fondateur, comme si tous les chemins menaient à Rome... Qu'est-ce qui est en jeu dans l'évidence d'une référence?

Dans ce qui suit nous présentons trois analyses de la matérialité linguistico-historique de noms de rue et ses conséquences pour les processus d'indentification du sujet urbainnational tant du point de vue de la construction des objets qu'il nomme que du point de vue de la compréhension qu'on peut avoir de ce sujet.

\section{Analyse}

\subsection{La période coloniale et l'invention de l'espace urbain}

Nous allons d'abord étudier le processus de nomination de la rue d'une église bâtie en Salvador (Bahia) dans le début de la colonisation du territoire brésilien. La construction d'églises fut une des premières préoccupations des Portugais dès que la politique d'occupation des terres brésiliennes se fut intensifiée (vers la 1530). L'établissement des villes avec des domiciles privés et des bâtiments publics - surtout des édifices destinés à l'administration politique (mairie) et religieuse (église) - faisait partie du projet colonisateur d'appropriation du pays et d'expulsion des peuples indigènes. Ce nouvel espace urbain devrait être bâti et aussi nommé avec l'espace européen comme référence. Cependant, ce modèle transposé prend un nouveau sens dans et par son installation dans un espace-temps autre. Nous défendons le point de vue admis par Pêcheux ${ }^{13}$ selon lequel il n'y a pas de domination sans résistance, la place occupée par le pouvoir fait du sens par les dominés à travers le processus d'identification et en même temps il est constamment déplacé par les pratiques sociales et langagières.

17 En ce qui concerne l'histoire de nomination de la rue analysée, on remarque que, officiellement, la partie de la voie qui passe devant l'Igreja de São Francisco reçoit le nom de Rua São Francisco et la partie qui passe devant l'Igreja da Ordem Terceira de São Francisco, s'appelle Rua da Ordem Terceira. Dans le premier cas, l'identification de la rue est une 
projection directe du nom du saint qui nomme aussi l'église et l'ordre religieux auquel elle appartient. Dans le deuxième, c'est l'ordre religieux même qui se caractérise en se disant tiers, c'est-à-dire soumis aux frères premiers, et qui établit, par l'intermède de la préposition de, un rapport entre le classificateur rue et le nom fonctionnant comme un individualisateur.

Il convient de souligner que physiquement ces deux rues sont contiguës; en regardant un plan ou en marchant dans cette rue, par exemple, on pourrait même dire qu'il ne s'agit que d'une seule rue, mais elle est toujours désignée par deux noms différents. Désormais, chacune des portions de la voie est identifiée comme un objet différent dans l'espace. La limite entre ces deux objets est entretenue dans l'énonciation officielle ${ }^{14}$ ainsi que dans les variations populaires dans le nom de la rue : dans une partie elle est connue comme Rua São Francisco, Rua da Igreja de São Francisco ou Rua do Cruzeiro de São Francisco et, dans l'autre, comme Rua da Ordem Terceira de São Francisco ou Rua dos Terceiros. La différence entre les deux rues ne se confond jamais. Les variations cohabitent dans le quotidien en réaffirmant une frontière toujours bien établie, de façon explicite ou elliptique, par le syntagme São Francisco.

stabilise la division de la rue par la remémoration d'une hiérarchie construite dans un autre espace urbain et qui raconte donc, en territoire brésilien, une histoire de la colonisation à travers le déplacement des institutions catholiques. Les noms de ces rues témoignent de la division de pouvoir à l'intérieur de l'église catholique et la préservation de ces mêmes noms pendant plus de quatre siècles projetant l'histoire religieuse européenne dans un espace national encore en formation.

Il est toutefois fondamental de considérer que la permanence de ces noms matérialise la transposition d'institutions d'un territoire déjà construit (l'Europe) par un territoire en construction (l'Amérique), mais ce déplacement n'est pas une simple imposition. Pour qu'il y ait de l'espace urbain, comme un effet d'unité du territoire, il faut que la transposition des institutions et ses noms fasse sens dans la nouvelle société. On peut dire que la naturalisation de la langue du colonisateur à travers sa diffusion comme langue nommant les espaces urbains constitue un instrument d'identification de l'espace urbain pour les citoyens qui y circulent.

\subsection{Les bases de l'indépendance}

21 Dans l'analyse suivante, nous nous pencherons sur un deuxième moment de l'histoire politique du Brésil, marqué par l'indépendance de la métropole, la consolidation du régime impérial et le développement consécutif des institutions proprement brésiliennes. L'Etat brésilien investit alors dans la construction de collèges et d'écoles en visant à l'éducation des élites nationales et à la formation d'un espace propre de production scientifique. A la fin du XIXe siècle, le Brésil discute déjà de la nécessité d'une rupture définitive avec la domination de la monarchie portugaise. La république apparaît aux yeux des grands propriétaires de terres brésiliens comme un moyen d'ascension au pouvoir. L'appropriation du positivisme français par cette élite conforte cette position. Ce cadre donne, grosso modo, une vue de la situation brésilienne au moment de la fondation du Collège Culture de la Science (1874) à Campinas. Inspirée de l'idéal positiviste, l'installation de ce collège va laisser ses vestiges dans l'espace urbain.

Avant l'existence de l'édifice du Collège Culture de la Science, la rue était appelée Rua Alegre: elle longeait des petites fermes et un espace de prostitution. La structure de cette 
appellation est donnée par l'adjectivation: la rue est reconnue comme rue gaie, possible rappel du commerce de plaisir qu'y si déroulait.

La construction du Collège dans cet espace est inscrite dans la nouvelle dénomination: Rua do Colégio. Prosaïquement on constitue un point de repère localisable. On peut même penser à un rapport d'appartenance : rue de quoi ? rue du collège. Il est intéressant de réfléchir sur les sens (moralisateurs) que l'ancien nom de la rue acquit dans les récits de fondation du collège : la motivation (a posteriori) du nom 'rue gaie' fut la gaieté des étudiants et non pas la possible gaieté d'une zone de prostitution.

Aujourd'hui, la rue est appelée Rua Culto à Ciência, nom qui représente de façon métonymique le passage de la mémoire de sa construction (rue du collège) à la mémoire de ce que l'on y enseigne, son système de valeur (la culture de la science) renvoyant au XIX ${ }^{\mathrm{e}}$ siècle et au positivisme d'Auguste Comte). Le rapport entre la rue et son nom est direct, transitif. Ainsi s'inscrit dans le corps de la ville l'appropriation brésilienne du positivisme de la science, de l'enseignement et de l'urbanité. La filiation à la scientificité française et sa réaffirmation dans l'espace urbain marquent une rupture avec les savoirs développés par le Portugal, ancienne métropole de laquelle la jeune nation brésilienne devait s'écarter pour construire un espace, une langue et un savoir-faire scientifique propres.

\subsection{Le spatialisation de la république}

Finalement, nous aborderons la période de consolidation de la république avec l'analyse de la construction, la dénomination et le changement du nom de l'avenue où est localisé le bâtiment actuel de la Bibliothèque Nationale du Brésil (début du XXe siècle).

L'Avenue Central au Rio de Janeiro est ouverte dans le début de XXème siècle comme un signe de modernité. C'est à ce moment que l'on construit un nouveau bâtiment pour la Bibliothèque Nationale du Brésil. Le nom Avenue Central soulève plusieurs questions au sujet du rapport langue-monde. D'abord, on peut dire que l'existence physique d'une voie urbaine ne va pas de soi, on a toujours besoin de dire, par le nom, ce qu'elle est. Le nom Avenue Central construit un événement linguistique où se marque le centre de la ville en même temps que se projette un futur qui désigne le rapport entre ce qui est le centre et ce qui est la périphérie.

En 1912 l'avenue change de nom pour rendre hommage au Baron de Rio Branco, décédé à cette année-là. Cela signifie fondamentalement un changement dans ce que l'avenue remémore : ce n'est plus la relation physique entre centre et périphérie, mais l'histoire politique de la ville. Linguistiquement, on peut décrire ce changement comme le déplacement d'un nom catégoriel (avenue) articulé syntaxiquement à un adjectif dérivé d'une locution adjective ( $c$ central $»=$ "du centre $)$ ) par un lien non prépositionnel ( avenue Rio Branco). On y repère l'installation d'une signalétique (Bosredon B. et Tamba I., 1999) motivée par un trait sémantique (l'avenue qui reste au centre, l'avenue la plus importante de la ville) qui, fondé sur l'hommage, n'assume qu'une des fonctions des relations dénominatives : celle de différencier un objet de l'autre, en le particularisant. Pour le dire autrement, il y a une différence entre s'appeler (x) parce que la rue a (ou elle eut) les caractéristiques de (x) et s'appeler (x) pour être simplement différenciée de (y).

Dans le cas étudié ici, la centralité de l'avenue n'est pas forcément un fait donné, elle est une construction de ce qu'était le centre du Rio de Janeiro dans ce moment de son urbanisation. En revanche, le statut de l'hommage, qui peut être conçu comme un geste 
de différenciation entre la rue $\mathrm{x}$ et la rue $\mathrm{y}$, peut être aussi pensé comme une partie importante de la construction d'une mémoire par la ville à partir de citoyens considérés illustres - dans le cas présent, un homme politique né à Rio de Janeiro qui représente soit la ville soit la nation dans ses rapports diplomatiques. Ce type de nomination produit une différence entre les citoyens: ceux qui méritent de donner leur nom aux espaces publiques et les autres. Comme relation de langage, la nomination est donc toujours une construction de sens qui identifie des objets pour les sujets. Selon Guimarães (2007: 82) cela se produit parce que les mots désignent, c'est-à-dire, signifient le réel historiquement dans l'événement de l'énonciation, c'est pourquoi il est possible de particulariser les référents.

Qu'il y ait ou non un rapport empiriquement motivé entre l'avenue et le Baron de Rio Branco ou entre l'avenue et le fait qu'elle soit centrale, on peut remarquer, du point de vue discursif, que ces noms de rues sont efficaces dans le geste de produire une mémoire de grandeur et d'héroïsme, références qui se projettent dans le quotidien et construisent la représentation des citoyens dans l'espace national.

\section{Conclusion : nommer l'espace et ses traces}

L'analyse du discours historique sur la nomination de l'espace urbain brésilien conduit à questionner la transparence de l'espace et l'effacement de son historicité. L'étude initiale de ces documents indique que la nomination de cet espace, construit et décrit, est une régularité thématique intéressante dans un corpus plus général qui raconte l'histoire du territoire brésilien. Du point de vue linguistique, on peut discuter les limites entre le nom commun et le nom propre et le rapport de sens d'un nom avec la construction du référent. Cette discussion touche toujours à la tension entre le linguistique et l'extralinguistique, la façon dont à partir du langage le monde se spécifie, se signifie.

31 Comme le rapport entre langage et monde n'est pas transparent, les noms projettent linguistiquement différents sens pour les objets nommés et, donc, par les sujets de langage. Il y a un imaginaire de nationalité en construction par la nomination de rues, imaginaire qui révèle la façon dont le Brésil signifie et légitime son propre événement comme unité imaginaire. En analysant ce petit parcours des noms de rues d'institutions qui sont devenues des références dans l'imaginaire national, on fait dialoguer le politique et l'historique avec le linguistique (Orlandi, 2001:21). On remarque que les expressions linguistiques ne s'attachent pas de façon univoque à leurs référents, mais la possibilité même de la référence est une construction du dire qui n'est pas en dehors de l'histoire. La référence, en tant que processus d'identification, fait partie du mouvement de signification du réel par le langage et par l'histoire.

En questionnant la sédimentation des savoirs que les constructions urbaines incarnent, nous avons tenté d'apporter une contribution à l'histoire des idées à travers la connaissance d'événements spécifiques qui peuvent montrer les mouvements de la pratique linguistique, urbanistique et historique dans la ville. Ce type d'étude permet de poser la compréhension de la production et de la circulation du savoir hors d'un point de vue purement institutionnalisé ; au contraire, interroger la mise en texte de savoirs dans la ville à travers les noms de rue c'est réfléchir aux conditions qui les rendent possibles dans les parcours institutionnels et quotidiens du langage par l'espace où ils circulent. Ce travail attribue donc à la ville comme une fonction d'auteur : ce qui est là en tant que matérialité urbaine est le résultat du conflit entre différents sens et une résistance 
silencieuse cachée dans le détail, au-dessous des couches de peinture, sous les façades de béton, derrière un nouveau nom ou dans le vide de la démolition...

\section{BIBLIOGRAPHIE}

AUROUX, S. (1995) La révolution technologique de la grammatisation. Liège : Mardaga.

ALTHUSSER, L. Réponse à John Lewis, Paris, Maspero, 1973.

BOSREDON, B. TAMBA I. (1999) Une ballade en toponymie : de la rue Descartes à la rue de Rennes, LINX 40, Université Paris 10, p. 55-69.

GUIMARÃES, E. (2005) Semântica do Acontecimento, Campinas : Pontes.

HAROCHE, C. Faire dire, vouloir dire. Presses universitaires de Lille, 1984.

HENRY, P. « L'histoire n'existe pas? » In: Studies in history of psychology and social sciences, 3. Roma: 1984.

MILNER, J.-C. (1978) L'amour de la langue. Lagrasse : Éditions Verdier, 2009.

ORLANDI, E. (2001) Discurso e Texto : formação e circulação dos sentidos. Campinas : Pontes.

ORLANDI, E. (org.). Política lingüística no Brasil. Campinas : Pontes, 2007.

ORLANDI, E. (org.). Un dialogue Atlantique : production des sciences du langage au Brésil. Lyon : ENS Editions, 2007a.

ORLANDI, E. Língua e conhecimento lingüístico. São Paulo : Cortez, 2002.

ORLANDI, E. e GUIMARÃES, E. (org.). Institucionalização dos estudos da linguagem. Campinas : Pontes, 2002a.

ORLANDI, E. (org.). Historia das idéias lingüísticas : construção do saber metalingüístico e constituição da língua nacional. Campinas : Pontes/UNEMAT, 2001a.

ORLANDI, E. (org.). Língua e Cidadania : o Português no Brasil. Campinas : Pontes, 1996.

ORLANDI, E. (org.) Cidade atravessada : os sentidos públicos no espaço urbano. Campinas : Pontes, $2001 b$.

ORLADI E. (2001c) « La ville comme espace politico-symbolique. Des paroles désorganisées au récit urbain », Langage et société 2001/2, n 96, p. 105-127.

ORLANDI, E. (org.) Para uma enciclopédia da cidade. Campinas : Pontes, 2003.

ORLANDI, E. Cidade dos sentidos. Campinas : Pontes, 2004.

ORLANDI, E. e RODRIGUES-ALCALÁ, C. «A produção do consenso nas políticas públicas urbanas : entre o administrativo e o jurídico » - CAeL. Projeto Temático Fapesp. In : Escritos no. 08, Labeurb/ Nudecri/ Unicamp, 2004a.

PÊCHEUX, M. (1975) Les vérités de la Palice, Paris, Maspero (coll. “Théorie"). Traduction brésilienne : Eni Orlandi [et al.] : Pêcheux M. Semântica e discurso : uma crítica à afirmação do óbvio. Campinas : Editora da Unicamp, 1995. 
PECHEUX, M. (1982) Délimitations, retournements et déplacements. In : L'homme et la société : revue internationale de recherches et de synthèses sociologiques, $\mathrm{n}^{\circ}$. 63-64, 1982, pages 53-70. Traduction brésilienne utilisée dans les citations : PECHEUX, M. « Delimitações, inversões, deslocamentos. » Cadernos de Estudos Lingüísticos no․ 19. Campinas : IEL/Unicamp, 1990.

PÊCHEUX, M. (1983) « Le discours : structure ou événement ? » In : MALDIDIER, D. (éd.) (1990) L'inquiétude du discours, Paris, Editions des Cendres, pp. 303 - 323. Traduction brésilienne utilisée dans les citations : Eni Orlandi. Pêcheux M. O discurso : estrutura ou acontecimento. $3^{a} \mathrm{ed}$. Campinas : Pontes, 2002.

Revista Rua. Revista do Laboratório de Estudos Urbanos Editée semestriellement depuis 1995. (Labeurb), Campinas : UNICAMP.

THIBAUD, J.-P. (2002) « L'horizon des ambiances urbaines. » In : Communications nำ 73, Paris : EHESS, 2002, p. $185-201$.

\section{NOTES}

1. On peut faire une différence entre les « inscriptions urbaines » : ce qui est du linguistique dans l'espace urbain (noms de voies et de lieux, tags, annonces, plaques, etc.) et les « entités urbaines » (les objets architecturaux de façon générale).

2. L'expression « forme-sujet » fut introduite par Althusser $(1973: 67)$ et fait référence à la forme d'existence historique de n'importe quel individu. La forme-sujet est différente de la situation sociale de l'individu. Tous les individus se soumettent au symbolique pour pouvoir dire, ce processus est appelé «processus de subjectivation » (Pêcheux, 1975), dont la langue et l'histoire participent activement.

3. Références bibliographiques importantes concernant le Projet Histoires des Idées Linguistiques au Brésil : Orlandi, E. 2007, 2007a, 2002, 2002a, 2001a, 1996.

4. Références bibliographiques importantes concernant l'axe de recherche Savoir urbain et langage : Revista Rua (depuis 1995) et Orlandi, E. 2001b, 2001c, 2003, 2004, 2004a.

5. Cf. Haroche, C. (1984).

6. Tout énoncé est susceptible de devenir un autre, différent de soi-même, de se déplacer discursivement de son sens et dériver par un autre. Le jeu métaphorique expose la langue à l'équivoque structurelle du symbolique. Pêcheux, M. (1983: 53 sq.).

7. Le mot « construction » est utilisé de façon polysémique (signifiant à la fois bâtiment, séquence linguistique et référent linguistique) - pour marquer la complexité de l'événement urbain et ses rapports aux phénomènes langagiers.

8. Evénement dans le sens donné par Pêcheux, M. (1983:20, 26, 27).

9. Orlandi, E. (2001: 21).

10. Le sens des mots nous semble évident, on oublie que le dire pourrait toujours avoir été formulé autrement, (Pêcheux, M., $1975: 190)$.

11. Milner, J.-C. (1978:64) et Pêcheux (1983: 56 et 57$)$.

12. Henry, P. (1984) et Pêcheux, M. ibidem.

13. Pêcheux, M. (1982: 16 et 17).

14. Par énonciation officielle j'entends les formes dénominatives utilisées par l'administration de la ville, recensées par la poste et indiquées sur les plaques de rue. 


\section{RÉSUMÉS}

En analysant les noms de rue de trois institutions brésiliennes, nous étudierons les liens entre le processus d'urbanisation et la légitimation du fait national au moyen de deux entrées : la langue, d'une part, et la circulation de savoirs historiques, culturels et philosophiques dans l'espace urbain, d'autre part. Parallèlement, nous analyserons la formation syntaxique des noms en posant la question du rapport entre la langue et la construction des objets nommés. Les objets urbains inscrits dans l'espace et leurs noms sont ici considérés comme des objets historiques dont l'étude peut apporter des contributions pour la compréhension d'événements spécifiques de la pratique langagière.

Researching street names of three Brazilian institutions, we study the relationship between their urbanization process and their native authenticity. Our bias of study is based on either the language, either the circulation of historical, cultural and philosophical issues concerning urban space. At the same time, we analyze the syntax quality of the names. We make it by comparing the possible affinities between language and names structure. This project strikes the importance of geographical and historical issues, leading to a superlative comprehension of the languages we use.

\section{INDEX}

Mots-clés : noms de rue, langue nationale, référence, discours urbain, Histoire, contexte

Keywords : history, native language, street names, urban discourse, context

\section{AUTEUR}

\section{CAROLINA FEDATTO}

Université Campinas, Brésil / Université Paris 3 - Sorbonne Nouvelle 